\title{
Surprising Intrinsic Photostability of the Disulfide Bridge Common in Proteins
}

\author{
Stephansen, Anne Boutrup; Brogaard, Rasmus Yding; Kuhlman, Thomas Scheby; Klein, Liv \\ Bærenholdt; Christensen, Jørn Bolstad; Sølling, Theis Ivan
}

Published in:

Journal of the American Chemical Society

Link to article, DOI:

10.1021/ja310540a

Publication date:

2012

Document Version

Publisher's PDF, also known as Version of record

Link back to DTU Orbit

Citation (APA):

Stephansen, A. B., Brogaard, R. Y., Kuhlman, T. S., Klein, L. B., Christensen, J. B., \& Sølling, T. I. (2012).

Surprising Intrinsic Photostability of the Disulfide Bridge Common in Proteins. Journal of the American Chemical Society, 134(50), 20279-20281. https://doi.org/10.1021/ja310540a

\section{General rights}

Copyright and moral rights for the publications made accessible in the public portal are retained by the authors and/or other copyright owners and it is a condition of accessing publications that users recognise and abide by the legal requirements associated with these rights.

- Users may download and print one copy of any publication from the public portal for the purpose of private study or research.

- You may not further distribute the material or use it for any profit-making activity or commercial gain

- You may freely distribute the URL identifying the publication in the public portal 


\title{
Surprising Intrinsic Photostability of the Disulfide Bridge Common in Proteins
}

\author{
Anne B. Stephansen, ${ }^{\dagger}$ Rasmus Y. Brogaard, ${ }^{\dagger}, \S$ Thomas S. Kuhlman, ${ }^{\dagger}$ Liv B. Klein, ${ }^{\dagger}$ Jørn B. Christensen, ${ }^{\dagger}$ \\ and Theis I. Sølling* ${ }^{\dagger}$
}

${ }^{\dagger}$ Department of Chemistry, University of Copenhagen, Universitetsparken 5, DK-2100 Copenhagen $\varnothing$, Denmark

${ }^{\ddagger}$ Department of Chemistry, Technical University of Denmark, Building 207, DK-2800 Kgs. Lyngby, Denmark

\section{Supporting Information}

ABSTRACT: For a molecule to survive evolution and to become a key building block in nature, photochemical stability is essential. The photolytically weak $S-S$ bond does not immediately seem to possess that ability. We mapped the real-time motion of the two sulfur radicals that result from disulfide photolysis on the femtosecond time scale and found the reason for the existence of the $S-S$ bridge as a natural building block in folded structures. The sulfur atoms will indeed move apart on the excited state but only to oscillate around the $S-S$ center of mass. At long $S-S$ distances, there is a strong coupling to the ground state, and the oscillatory motion enables the molecules to continuously revisit that particular region of the potential energy surface. When a structural feature such as a ring prevents the sulfur radicals from flying apart and thus assures a sufficient residence time in the active region of the potential energy surface, the electronic energy is converted into less harmful vibrational energy, thereby restoring the $\mathrm{S}-\mathrm{S}$ bond in the ground state.

$\mathrm{T}$ he key chemical cornerstones of nature are the DNA nucleobases and the amino acids. These molecules have survived a natural selection since the beginning of time because they are particularly well suited to resist exposure to heat and sunlight. In the case of the DNA bases it has been shown that there is a particularly favorable pathway for disposing of the electronic energy in an electronically excited state by converting it into ground-state vibrational energy that can easily be dissipated to the surroundings by thermalization to become harmless. ${ }^{1}$ The mechanism involves efficient coupling of the ground and excited electronic states through a subset of the available degrees of freedom via a conical intersection-the socalled photochemical funnel. ${ }^{2,3}$ In the case of the DNA bases, this subset includes the degrees of freedom that become activated in the absorption process, and this is what opens the possibility for an ultrafast repopulation of the ground state. Similar effects are in play for the amino acids and thus also generally in proteins. ${ }^{4}$ For DNA and proteins the threedimensional structure is crucial in determining the molecular function: DNA has its double helix, and a protein its tertiary structure. We have set out to investigate what structural elements have enabled the three-dimensional structure to survive in the form that it has. The disulfide bridge is one of the central building blocks in nature, and it is an important factor in the determination of tertiary structure in proteins. ${ }^{5}$ At first sight this does not agree very well with what else is known about the stability and particularly the photochemistry of disulfides. Already very early on, it was well recognized that disulfides, upon the absorption of light, cleave to form free radicals which take part in various chemical processes rather than re-forming the S-S bond. ${ }^{6}$ Time-resolved studies of the $S-S$ bond breakage showed that the rupture does indeed take place on the sub-picosecond time scale. ${ }^{7}$ An ultrafast $S-S$ bond breakage does not speak in favor of the $S-S$ bond as a key driver in determining protein structure because it simply would not have survived intense UV radiation since the beginning of time. So what has saved the $\mathrm{S}-\mathrm{S}$ structural element? We believe that it is the confinement that is built into the cyclic arrangement of the tertiary structure in proteins. The $\mathrm{S}-\mathrm{S}$ bond can be perturbed by light, but the sulfur atoms are restricted so that they cannot move apart. This type of behavior is exactly what has been found for solvated disulfides exposed to UV radiation, where it has been argued that the nascent radicals are captured in a solvent cage and therefore cannot escape. ${ }^{7-9}$ This caging effect will decrease the quantum yield of radical formation, but in some biologically relevant systems a $30 \%$ yield is still obtained in the absence of intrinsic structural constraints. ${ }^{10} \mathrm{We}$ have focused on the photoinduced dynamics of dithiane (Scheme 1), which is the smallest possible saturated cyclic structure containing an $S-S$ bond that is stable enough to be exposed to ambient experimental conditions.

Scheme 1. Photolytic Cleavage of S-S Bond in Dithiane

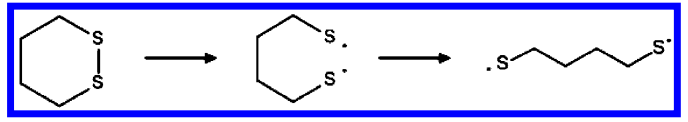

This compound mimics the structural motif involving the S$S$ bond that stabilizes the tertiary and quaternary structure in many proteins. We have conducted pump-probe experiments in a femtosecond time-resolved mass spectrometry (TRMS) apparatus on a molecular beam that is generated in a supersonic expansion. Dithiane was excited to the $S_{1}$ state by $284 \mathrm{~nm}$ pulses with a time duration of $110 \mathrm{fs}$, while a $400 \mathrm{~nm}$ femtosecond probe pulse ionized the excited-state population and the temporal evolution of the resulting ion current was

Received: October 25, 2012

Published: December 4, 2012 
measured. The experiment was conducted in the gas phase to fully eliminate the effects of solvent enclosure of the product radicals and thereby to get the full picture of the intrinsic dynamics of cyclic structures containing the $S-S$ bond.

Our experiments have been backed up by a series of ab initio calculations. The calculated energy change as a function of the $\mathrm{S}-\mathrm{S}$ distance is shown in Figure 1. There are no barriers

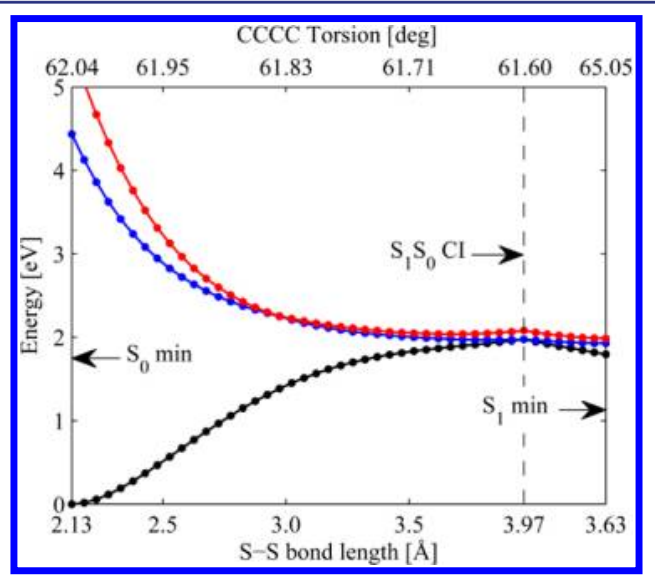

Figure 1. Potential energy surfaces of the three lowest singlet excited states of dithiane calculated at the SA-3-CAS $(10,8) / 6-31 \mathrm{G}(\mathrm{d}, \mathrm{p})$ level of theory. The structures have been linearly interpolated in internal coordinates from the $S_{0}$ minimum to the $S_{0} S_{1}$ conical intersection and from the latter to the $S_{1}$ minimum.

exhibited along the way, and the route from the FranckCondon geometry to a transient species with a very long $S-S$ bond is steeply downhill on the first excited state. This means that the sulfur atoms experience a large force pushing them away from each other, and if there was nothing to hold them back, they would fly apart immediately. Looking further at longer $S-S$ distances, it is possible to characterize an $S_{1}$ state minimum geometry and a minimum-energy conical intersection point that connects the $S_{1}$ and $S_{0}$ states (Figures 1 and 2). The

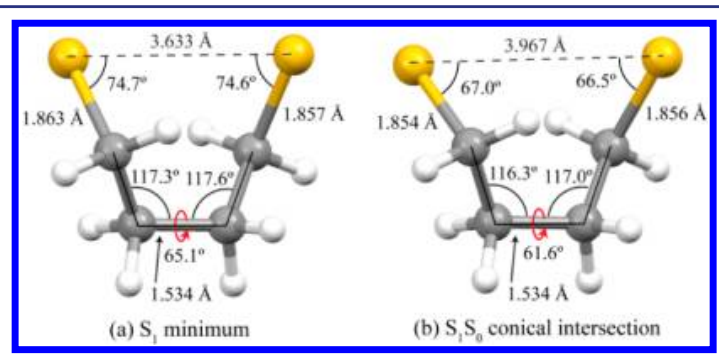

Figure 2. Optimized geometries (SA-3-CAS $(10,8) / 6-31 G(d, p)$ ) of dithiane for (a) the $S_{1}$ minimum and (b) the minimum-energy $S_{1} S_{0}$ conical intersection. Note that only small changes in angles and bond lengths will transform one into the other.

calculated geometry at the conical intersection point closely resembles that of the $S_{1}$ minimum species: the two points on the potential energy surface are connected simply by a slight torsion of a $-\mathrm{CH}_{2} \mathrm{~S}^{\bullet}$ group. Thus, once the molecules start to move downhill on the potential energy landscape toward the $S_{1}$ minimum, the region near the conical intersection will also be visited. Given enough time in this coupling region of the potential energy surface, the nascent $S_{1} S-S$ diradical will end up on the ground-state surface to re-form the $S-S$ bond, and thus the initial disulfide will prevail.
The key finding of this report is that the cyclic structure provides the necessary lifetime, because the time scale for funneling the internal energy into the degrees of freedom that could result in unfolding the carbon chain to move the molecule away from the active region is picosecondstraditionally referred to as the time scale of intramolecular vibrational energy redistribution (IVR). ${ }^{11}$ The dynamics of this process is illustrated in Figure 3. Upon excitation to the $S_{1}$ state

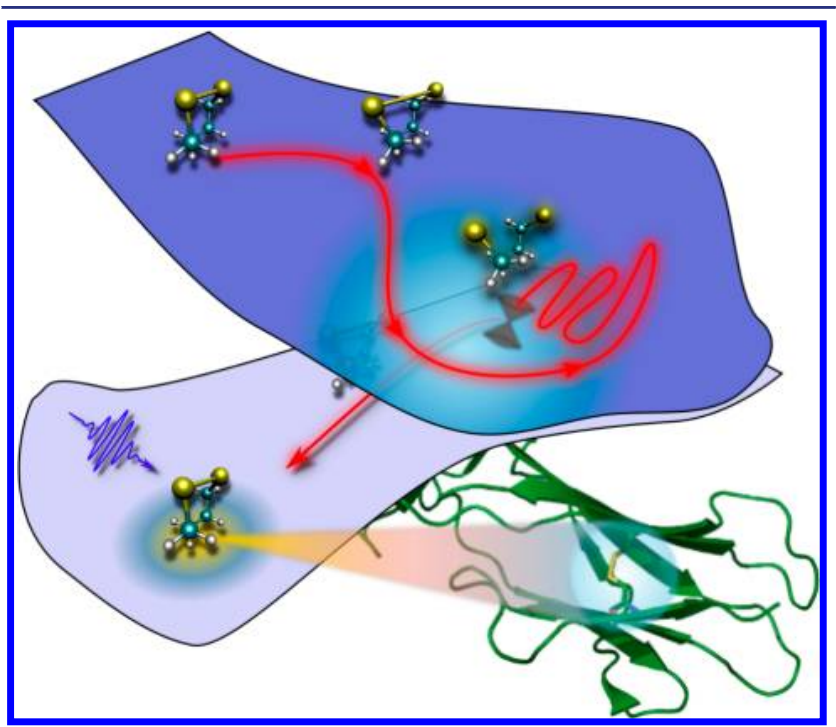

Figure 3. General picture of how an $\mathrm{S}-\mathrm{S}$ bond in a cyclic structure is deactivated. Subsequent to excitation the molecules stretch the $S-S$ bond to start a torsional motion along the carbon backbone; this dynamical behavior makes the molecule visit the region of the conical intersection where a strong interaction connects $S_{1}$ with $S_{0}$. Once on the ground state, the $S-S$ bond is re-formed.

the $S-S$ bond stretches, and the stretch is accompanied by a wiggling motion of the carbon backbone that takes the sulfur atoms back and forth past each other. During this motion, an electronic transition to the ground state takes place, resulting in ground-state dithiane molecules with an intact $S-S$ bond.

The TRMS experiment beautifully reveals the dynamics in the time-resolved ion current shown in Figure 4 together with a fit of the signal. The initial ultrafast decay corresponds to the $S-S$ bond stretch and reveals that the $S_{1}$ minimum is reached in $<200 \mathrm{fs}$. It is on this time scale that a straight chain RS-SR disulfide will form two $\mathrm{R}-\mathrm{S}^{\bullet}$ radicals. Intriguingly, this is prevented by the wiggling motion that is observed as a periodic modulation of the signal; this is as direct a marker of molecular motion as possible in all UV-vis pump-probe experiments. The modulation comes about since the transient diradicaloid species are easier to ionize when the sulfur atoms are close to each other, because in this case the positive charge on the one sulfur atom can be stabilized by the lone pair on the other (Figure 4). Thus, the result is a peak in the signal when the sulfur atoms are in proximity and a valley when they are apart.

The time-resolved data are in complete agreement with the quantum mechanical calculations in that the calculated period of the wiggling motion in the $S_{1}$ excited diradicaloid species of $416 \mathrm{fs}$ is identical to the value of $411 \pm 27 \mathrm{fs}$ obtained from the fit to the experimental data. We note that this motion is mainly a torsion of a $-\mathrm{CH}_{2} \mathrm{~S}^{\bullet}$ group, ${ }^{12}$ exactly the motion that will take the molecule back and forth between the $S_{1}$ minimum and the conical intersection, thereby facilitating the electronic transition 


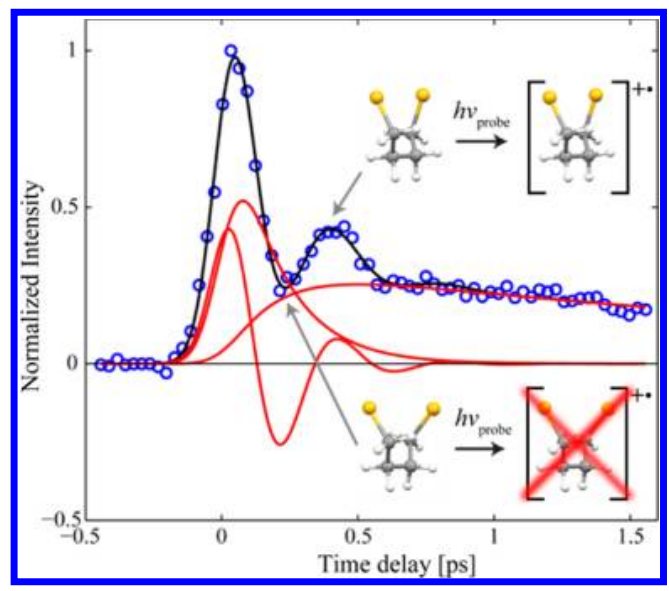

Figure 4. Temporal evolution of the ion current for the $\mathrm{m} / z 55$ fragment ion of dithiane. Experimental data are given by points, while the fitted signal is given by the black line. Also shown in red are the different components of the fit: two exponential decays and a damped oscillation. As indicated by the inserted structures, the oscillatory modulation of the ion current is due to the change in the ionization cross section as the molecule wiggles.

to the ground state. The transition is visible in the second decay component of the signal, revealing an excited-state lifetime of $2.75 \pm 0.23 \mathrm{ps}$, and it is apparent as a decaying ion current because the ground-state species cannot be ionized by the probe. The relatively long lifetime can be understood by considering that the conical intersection is sloped ${ }^{13}$ and situated uphill from the $S_{1}$ minimum. ${ }^{14}$ As such, the mechanism preserving the $S-S$ bond is significantly slower than the one protecting DNA from photodamage. Nevertheless, it is in both cases non-ergodic internal conversion that is key; here the mechanism is to salvage the spatial structure of proteins by populating the ground state in the initial molecular form faster than the dissipation of energy to the molecular degrees of freedom that will lead to unfolding of the ring. In DNA it is to populate the ground state faster than excited-state reactions can happen. The protection mechanism in DNA is induced by ultrafast motion of a proton in a Watson-Crick base pair and loss of planarity resulting in a sub-picosecond excited-state lifetime. ${ }^{15,16}$ In the $S-S$ structural motif, the large-amplitude wiggling motion is significantly slower, and the lifetime increases to a few picoseconds. In both cases the motions mediating a safe return to the ground electronic state are initiated at the time of light absorption; it seems that nature is indeed trying to teach us a few tricks in designing photostable molecular structures and photostable spatial arrangements.

\section{ASSOCIATED CONTENT}

\section{S Supporting Information}

Details of the experimental setup, electronic structure calculations, and method of fitting the experimental data. This material is available free of charge via the Internet at http://pubs.acs.org.

\section{AUTHOR INFORMATION}

\section{Corresponding Author}

theis@kiku.dk

\section{Present Address}

${ }^{\S}$ Department of Chemical Engineering, Stanford University, Stanford, CA 94305, USA

\section{Notes}

The authors declare no competing financial interest.

\section{ACKNOWLEDGMENTS}

The Danish Center for Scientific Computing (DCSC) is acknowledged for computational resources.

\section{REFERENCES}

(1) Crespo-Hernandez, C. E.; Cohen, B.; Kohler, B. Nature 2005, 436, $1141-1144$.

(2) Crespo-Hernandez, C. E.; Cohen, B.; Kohler, B. Nature 2006, 441, E8.

(3) Nielsen, S. B.; Sølling, T. I. ChemPhysChem 2005, 6, 1276-1281.

(4) Tomasello, G.; Wohlgemuth, M.; Petersen, J.; Mitric, R. J. Phys. Chem. B 2012, 116, 8762-8770.

(5) Creighton, T. E. BioEssavs 1988, 8, 57-63.

(6) Lyons, W. E. Nature 1948, 162, 1004.

(7) Ernsting, N. P. Chem. Phys. Lett. 1990, 166, 221-226.

(8) Hirata, Y.; Niga, Y.; Makita, S.; Okada, T. J. Phys. Chem. A 1997, 101, 561-565.

(9) Bultmann, T.; Ernsting, N. P. L. Phvs. Chem. 1996, 100, 1941719424.

(10) Milanesi, L.; Reid, G. D.; Beddard, G. S.; Hunter, C. A.; Waltho, J. P. Chem.-Eur. I. 2004, 10, 1705-1710.

(11) Diau, E. W.-G.; Herek, J. L.; Kim, Z. H.; Zewail, A. Science 1998, 279, 847-851.

(12) See Supporting Information Figure S3.

(13) Martinez, T. I. Nature 2010, 467, 412-413.

(14) See Supporting Information Figure S2 and the characterization therein.

(15) Sobolewski, A. L.; Domcke, W. Phys. Chem. Chem. Phys. 2004, 6, 2763-2771.

(16) Middleton, C. T.; de La Harpe, K.; Su, C.; Law, Y. K.; CrespoHernandez, C. E.; Kohler, B. Annu. Rev. Phys. Chem. 2009, 60, 217239. 